\title{
Synthesis of 2-Hydroxy-3-isopropoxypropyl Guar Gum and Its Thermo-responsive Property for Controlled Release
}

\author{
Ye Tian, a,b,* Yue Shang, ${ }^{\mathrm{a}, \mathrm{b}} \mathrm{He} \mathrm{Ma},{ }^{\mathrm{a}, \mathrm{b}}$ and Ying Liu ${ }^{\mathrm{a}, \mathrm{b}, *}$ \\ 2-Hydroxy-3-isopropoxypropyl guar gum (HIPGG), which is a novel \\ polysaccharide-based thermo-responsive polymer, was synthesized via \\ grafting a hydrophobic reagent (isopropyl glycidyl ether (IPGE)) onto a \\ hydrophilic main backbone (guar gum (GG)). The HIPGG exhibited \\ reversible and tunable thermo-responsive properties. Changing the molar \\ substitution (MS) of the hydrophobic side chain enabled the lower critical \\ solution temperature (LCST) to be adjusted within the range of $29.6^{\circ} \mathrm{C}$ to \\ $43.7^{\circ} \mathrm{C}$. Fluorescence spectrometry, dynamic light scattering (DLS), and \\ transmission electron microscopy (TEM) were used to investigate the self- \\ assembly behavior of HIPGG and the thermo-dependent size of its \\ aggregates. It was demonstrated that HIPGG formed stable aggregates in \\ aqueous solution, and the diameters of the aggregates increased as \\ temperature increased. Subsequently, Nile red was used as a model to \\ investigate the encapsulation and temperature-controlled release \\ behaviors in HIPGG aggregates. The results indicated that Nile red was \\ easily encapsulated in the hydrophobic region of HIPGG aggregates, and \\ its release at $36{ }^{\circ} \mathrm{C}, 38^{\circ} \mathrm{C}$, and $42{ }^{\circ} \mathrm{C}$ revealed that temperature had a \\ remarkable impact on release behavior.
}

Keywords: Thermo-responsive; Guar gum; Aggregates; Temperature-controlled release

Contact information: a: College of Marine Technology and Environment, Dalian Ocean University, Dalian 116023, China; b: Key Laboratory of Environment Controlled Aquaculture, Ministry of Education, Dalian Ocean University, Dalian, 116023, China;

*Corresponding authors: tianye@dlou.edu.cn; yingliu@dlou.edu.cn

\section{INTRODUCTION}

Stimuli-responsive polymeric materials are a new class of materials that can change their physicochemical properties in response to external environmental factors, such as temperature, $\mathrm{pH}$, ionic strength, and light intensity (Wu et al. 2016; Alejo et al. 2019; Yuba 2020). In recent years, stimuli-responsive polymers that have amphiphilic structures have received widespread attention in the field of biological medicine for two main reasons. First, these stimuli-responsive polymers self-assemble to form nanometer-sized aggregates or micelles that can be used as nanocarriers. Second, these micelles or aggregates can reversibly release or load encapsulated molecules under stimulation by external factors (Grzelczak et al. 2019).

Among these types of stimuli-responsive polymers, thermo-responsive polymers are the most intensively researched because their temperature changes are relatively easy to achieve and control. These polymers can also be conveniently and safely used in applications such as bio-separation, controlled release, and smart bioactive surfaces (Roy et al. 2013). Poly(N-isopropylacrylamide) (PINPAM) and its derivatives have been the most common thermo-responsive polymers. In aqueous solutions, PINPAM has a lower 
critical solution temperature (LCST) that is close to the temperature of the human body. Thus, PINPAM has received widespread attention in the fields of biomedicine (Carter et al. 2006). PNIPAAM-based polymers can self-assemble into micelles that encapsulate drugs when the temperature is less than the LCST. In contrast, micelles are destabilized, and drugs are quickly released when the temperature is greater than the LCST. Obviously, the LCST is the most important parameter for thermo-responsive polymers, and accurate control of the LCST allows the thermo-responsive polymers to be used in different fields. Tuning the LCST of PNIPAAM-based polymers is generally performed via the addition of inorganic salts and solvents or copolymerization with other hydrophobic or hydrophilic monomers (Chen and Guan 2004; Nagase et al. 2018; Bordat et al. 2019).

In the past few decades, polysaccharide-based thermo-responsive polymers have been extensively researched because polysaccharides are naturally nontoxic, biocompatible, and biodegradable (Shen and Patel 2008). Typically, polysaccharide-based thermo-responsive materials can be prepared via the graft copolymerization of polysaccharides with synthetic thermo-responsive polymers (grafting-to) or monomers (grafting-from) (Graham et al. 2019). Guar gum (GG) is a biodegradable and biocompatible gum that is extracted from guar beans, and its main component is galactomannan, which is hydrophilic. In addition, the guar structure contains many highly reactive hydroxyl groups, which allow other chemical modifications to occur (Prabaharan 2011; Gupta et al. 2015; Wang et al. 2018). Due to these advantages, GG seems to be an ideal candidate for preparing thermo-responsive materials. Therefore, the grafting-to approach has been used to graft thermo-responsive polymers onto GG. GG-based thermoresponsive copolymers can be prepared via the grafting of semitelechelic PNIPAM or poly(ethyleneoxide-co-propylene oxide) onto a backbone of GG derivatives, and the LCST of copolymers is in accordance with that of synthetic thermo-responsive polymers (Gupta et al. 2011, 2015). In other words, the synthetic thermo-responsive polymer moieties control the LCST of these copolymers. Further, both copolymers can form micelles or aggregates in aqueous solution. However, these thermo-responsive copolymers have their own limitations (Atanase et al. 2017; Graham et al. 2019). First, it is difficult to tune their LCST because the methods for changing the thermo-responsive nature of a synthetic polymer are complex, and this limits their practical applications in different fields. Second, the use PNIPAM and other synthetic polymers in biomedical applications is problematic, as their monomers are toxic to humans, and they are not biodegradable. Because of these factors, it is of great interest to modify polysaccharides with nontoxic small molecules and achieve thermo-responsive behavior via controlling the balance between the hydrophilic and hydrophobic groups in polysaccharide-based polymers. In previous studies, a series of novel thermo-responsive cellulose-based polymers were synthesized via the grafting of hydrophobic short alkyl chains onto cellulose, and their LCSTs ranged from $17.1{ }^{\circ} \mathrm{C}$ to $56.1{ }^{\circ} \mathrm{C}$. These amphiphilic polymers can also form micelles or aggregates when their concentration is above the critical aggregation concentration (CAC) (Tian et al. 2016; Dai et al. 2019a, 2019b).

In this study, a novel polysaccharide-based thermo-responsive polymer, namely 2hydroxy-3-isopropoxypropyl guar gum (HIPGG) is reported. It was produced via the grafting of isopropyl glycidyl ether (IPGE) onto GG using an etherification reaction. Indeed, HIPGG exhibited reversible thermo-responsive behavior, and changing the molar substitution (MS) of the hydrophobic side chain and the salt concentrations could be used to tune LCST values. Further, HIPGG had an amphiphilic structure and could selfassemble into aggregates in aqueous solution, and the thermo-responsive behavior of the 
HIPGG aggregates was investigated. Nile red was used as a model hydrophobic guest molecule, and the encapsulation and release behaviors of Nile red were investigated under different temperatures.

\section{EXPERIMENTAL}

\section{Materials}

The GG was purchased from Sigma-Aldrich (Saint Louis, MO, USA). Isopropyl glycidyl ether (IPGE) (> 96\%) was purchased from Tokyo Chemical Industry Co., Ltd. (Tokyo, Japan). Pyrene and 9-diethylamino-5H-benzo[a]phenoxa-]phenoxazine-5-one (Nile red) were supplied by J\&K Chemical, Ltd. (Beijing, China). Sodium chloride ( $\mathrm{NaCl}$, analytical reagent) and sodium hydroxide $(\mathrm{NaOH}$, analytical reagent) were purchased from Damao Chemical Reagent Factory (Tian Jing, China). Sodium chloride, sodium carbonate, and sodium thiocyanate were purchased from Aladdin Industrial Corporation (Shanghai, China). All the chemical reagents were used as received without further treatment.

\section{Preparation of 2-Hydroxy-3-isopropoxypropyl Guar Gum}

The GG $(1.0 \mathrm{~g})$, deionized water $(10 \mathrm{~mL})$, and sodium hydroxide solution $(1.6 \mathrm{~g}$, $40 \%$ ) were added to a $100-\mathrm{mL}$ three-necked flask. The mixture was stirred and heated to $70{ }^{\circ} \mathrm{C}$, and alkalization then proceeded for $1 \mathrm{~h}$. IPGE $(2.1 \mathrm{~g}, 2.5 \mathrm{~g}, 2.9 \mathrm{~g}, 3.3 \mathrm{~g}, 3.7 \mathrm{~g})$ was then slowly added dropwise, and the temperature was increased to $80{ }^{\circ} \mathrm{C}$. The reaction proceeded for $6 \mathrm{~h}$. After reaction, the system was cooled to room temperature, and $1 \mathrm{M}$ acetic acid was used to neutralize the solution to a $\mathrm{pH}$ of 7 . The mixed solution was dialyzed for $72 \mathrm{~h}$ in a dialysis bag with a molecular weight cutoff of $8000 \mathrm{Da}$ to $14000 \mathrm{Da}$. Most of the water in the resulting solution was removed using a rotary evaporator, and the remaining water was removed using a freeze-dryer (Biocool FD-1-135, Boyikang Experimental Instrument Co. LTD, Beijing, China) to obtain dried HIPGG.

\section{Methods}

The ${ }^{1} \mathrm{H}$-NMR ( ${ }^{1} \mathrm{H}$-nuclear magnetic resonance) spectra of HIPGG samples in $\mathrm{D}_{2} \mathrm{O}$ were recorded on a Varian INOVA 500 spectrometer (Palo Alto, CA, USA). Transmittance at $\lambda=590 \mathrm{~nm}$ was monitored using a Mettler Toledo T90 (Zurich, Switzerland) with a temperature controller (LAUDA RP200) and a heating rate of $1{ }^{\circ} \mathrm{C} / \mathrm{min}$ to determine the LCST values of the HIPGG solution. The LCST values were determined as the temperature at which the transmittance of the samples was around 50\% relative to its original value.

Transmission electron microscopy (TEM, FEI TF30; FEI Company, Hillsboro, OR, USA) with an accelerating voltage of $80 \mathrm{kV}$ was used to obtain images for analyzing the morphologies of HIPGG aggregates. The samples of HIPGG aggregates at temperatures below or above the LCST were prepared using a procedure similar to previously reported procedures (Tian et al. 2016). The dynamic light scattering (DLS) experiments were performed using a MalvernNano-ZS90 (Malvern Instruments, Malvern, England) with a $\mathrm{He}-\mathrm{Ne}$ laser operated at $632 \mathrm{~nm}$. The concentration of the samples was $1 \mathrm{~g} / \mathrm{L}$, and the samples were filtered using a filter with a pore diameter of $450 \mathrm{~nm}$ before measurement. The DLS measurements of the sample solutions were taken at temperatures in the range of $20{ }^{\circ} \mathrm{C}$ to $50{ }^{\circ} \mathrm{C}$ with a heating rate of $2{ }^{\circ} \mathrm{C} / \mathrm{min}$. 


\section{Determination of the CAC of HIPGG}

A fluorescence spectrometer (Hitachi F7000; Hitachi Ltd., Tokyo, Japan) was used to measure the CAC of HIPGG. To use pyrene as a fluorescence probe, a syringe pump was used to add $10 \mu \mathrm{L}$ of pyrene in acetone to a $10-\mathrm{mL}$ volumetric flask. The solution was heated at $50{ }^{\circ} \mathrm{C}$ for $2 \mathrm{~h}$ to dry the acetone. Then, the HIPGG solution was added, and the flask was sonicated to dissolve the sample. The solution was then allowed to stand overnight. The measurement conditions were as follows: an emission wavelength of 373 $\mathrm{nm}$, a scanning range of $305 \mathrm{~nm}$ to $365 \mathrm{~nm}$, and a measurement temperature of $20^{\circ} \mathrm{C}$.

\section{Encapsulation and Release of Nile Red}

A fluorescence spectrometer (Hitachi F7000) with an excitation wavelength of 490 $\mathrm{nm}$ was used to determine the behaviors of encapsulation and temperature-controlled release of Nile red. The preparation of each aqueous solution of HIPGG with a constant concentration of Nile red was carried out according to the procedure described in previous literature (Kim et al. 2009; Park et al. 2010; Tian et al. 2016). Nile red in a solution of acetone was added to a $10-\mathrm{mL}$ volumetric flask, and the acetone was removed via evaporation. Aqueous solutions with different concentrations of HIPGG were each loaded into a volumetric flask and sonicated in an ice bath for $4 \mathrm{~h}$. Then, the solutions stood overnight. The final Nile red concentration in each aqueous solution of HIPGG samples was $20 \mathrm{mg} / \mathrm{L}$. All samples were filtered using a 450-nm filter before measurement. In the temperature-controlled release experiment of Nile red, the samples were maintained at 36 ${ }^{\circ} \mathrm{C}, 38{ }^{\circ} \mathrm{C}$, or $42{ }^{\circ} \mathrm{C}$. Then, the samples were used to investigate the release behaviors under these temperatures, and each sample was monitored for three independent measuring results from which average values were obtained.

\section{RESULTS AND DISCUSSION}

\section{Synthesis and Characterization of HIPGG}

The synthetic route for HIPGG is shown in Fig. 1. The main chain GG is hydrophilic and reacted with the hydrophobic reagent (IPGE). Adjusting the amount of hydrophobic reagent enabled HIPGG with different MS to be obtained. Table 1 illustrates the characteristic parameters of HIPGG with different MS and the thermo-responsive properties of its aqueous solutions.

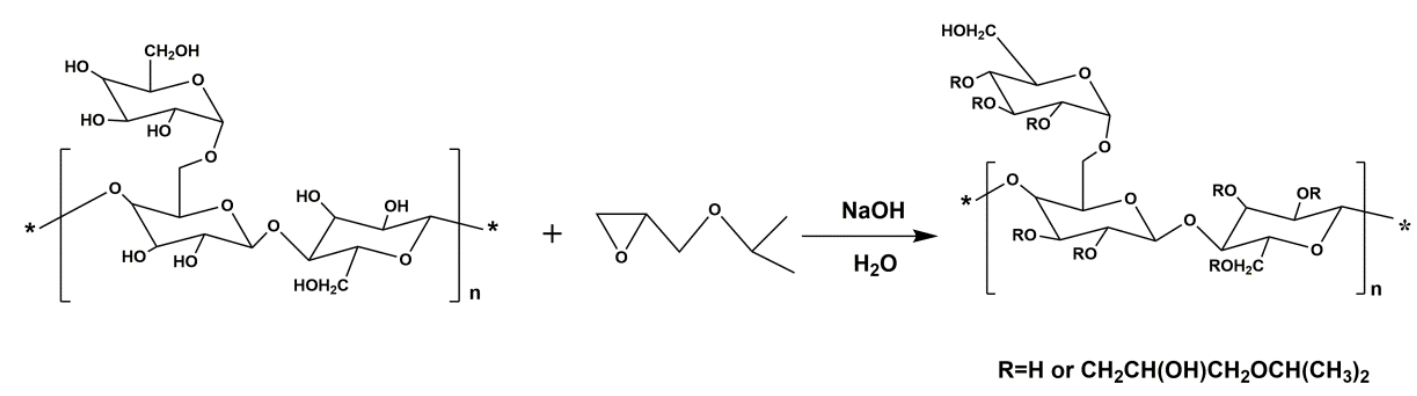

Fig. 1. Synthetic route for HIPGG

Figure 2 shows the ${ }^{1} \mathrm{H}-\mathrm{NMR}$ spectra of HIPGG. The signals at $1.08 \mathrm{ppm}$ were characteristic of the isopropyl group ( $\mathrm{H} 8)$. The signals at $5.30 \mathrm{ppm}$ to $5.80 \mathrm{ppm}$ were ascribed to the anomeric protons (H1) of HIPGG-3, and these indicated partial substitution 
at the hydroxyl of the O-2 position. The broader signal between $3.30 \mathrm{ppm}$ and $4.40 \mathrm{ppm}$ corresponded to protons of the anhydroglucose units and protons of the $\mathrm{O}-\mathrm{CH}_{2}-\mathrm{CHOH}-$ $\mathrm{CH}_{2}-\mathrm{O}-\mathrm{CH}_{2}$ group. The MS of HIPGG was expressed using the ratio of the integrated peak area of the methyl in the isopropyl group to six times the integrated peak area of H1 in the anhydroglucose unit (AGU). These results indicated successful etherification.

Table 1. Preparation and Characterization of HIPGG

\begin{tabular}{|c|c|c|c|c|}
\hline Sample & $\mathrm{n}(\mathrm{IPGE}): \mathrm{n}(\text { AGU) })^{\mathrm{a}}$ & $\mathrm{MS}^{\mathrm{b}}$ & ${\text { LCST }\left({ }^{\circ} \mathrm{C}\right)^{\mathrm{c}}}$ & $\mathrm{CAC}^{\text {at } 2{ }^{\circ} \mathrm{C}(\mathrm{g} / \mathrm{L})^{\mathrm{d}}}$ \\
\hline HIPGG-1 & 3.0 & $0.98 \pm 0.02$ & $43.7 \pm 1.7$ & $0.108 \pm 0.012$ \\
\hline HIPGG-2 & 3.5 & $1.19 \pm 0.03$ & $41.2 \pm 1.2$ & $0.071 \pm 0.008$ \\
\hline HIPGG-3 & 4.0 & $1.42 \pm 0.04$ & $37.1 \pm 1.0$ & $0.051 \pm 0.006$ \\
\hline HIPGG-4 & 4.5 & $1.63 \pm 0.01$ & $34.1 \pm 1.4$ & $0.046 \pm 0.009$ \\
\hline HIPGG-5 & 5.0 & $2.01 \pm 0.05$ & $29.6 \pm 1.1$ & $0.031 \pm 0.005$ \\
\hline
\end{tabular}

aMole ratio of etherifying agent to glucose units of guar gum

${ }^{b} \mathrm{MS}$ was determined by ${ }^{1} \mathrm{H}-\mathrm{NMR}$

'Determined via a Mettler Toledo T90 with a LAUDA RP200 temperature controller

${ }^{d} C A C$ was determined via a Hitachi F7000 fluorescence spectrometer

IPGE: Isopropyl glycidyl ether; AGU: Anhydroglucose units; MS: Molar substitution; LCST: Lower critical solution temperature; HIPGG: 2-Hydroxy-3-isopropoxypropyl guar gum

Values of MS, LCST, and CAC are mean of three experiments \pm SD.

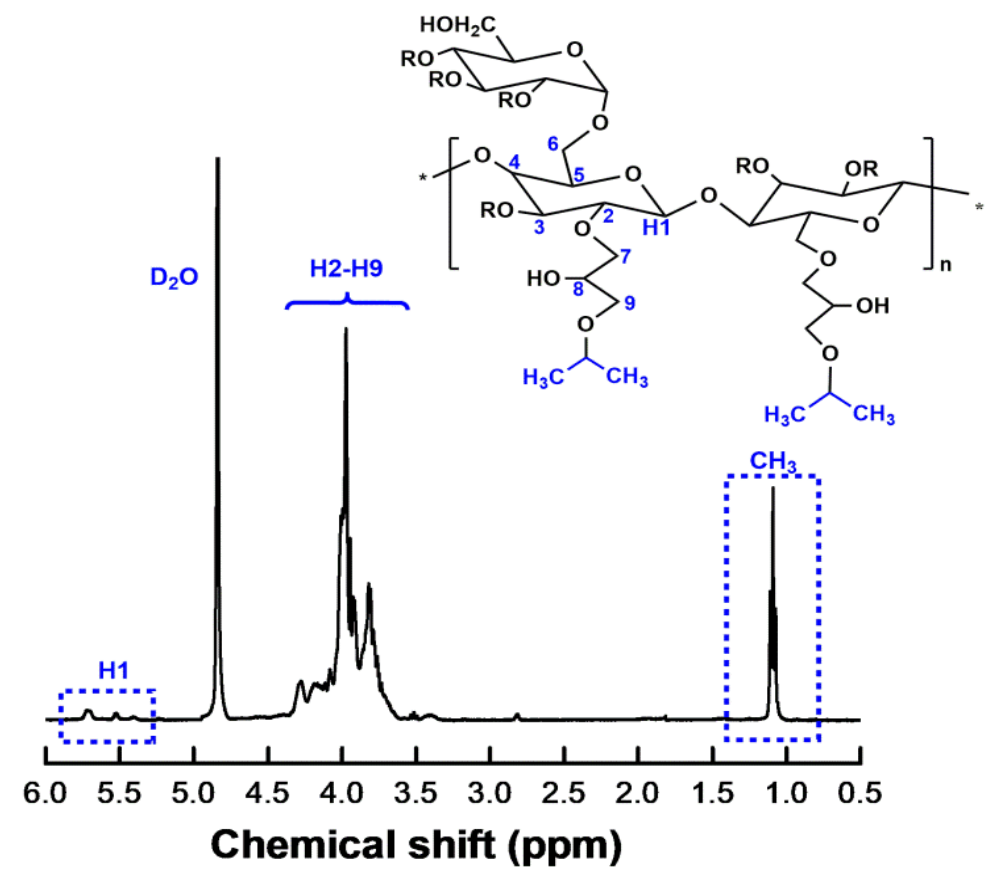

Fig. 2. The ${ }^{1} \mathrm{H}-\mathrm{NMR}$ spectra of $\mathrm{HIPGG}-3$ recorded in $\mathrm{D}_{2} \mathrm{O}$

\section{Thermo-responsive Behavior of HIPGG Solutions}

The LCST is among the most important parameters for describing thermoresponsive properties of polymers. Figure 3 a shows that the samples of aqueous solutions of HIPGG were transparent below their LCST, and the solution became turbid when the temperature increased above the LCST. When the temperature was decreased, the solution became transparent again. This indicated that the thermo-responsive phase-separation behavior of the HIPGG aqueous solution was reversible in nature. 
(a)

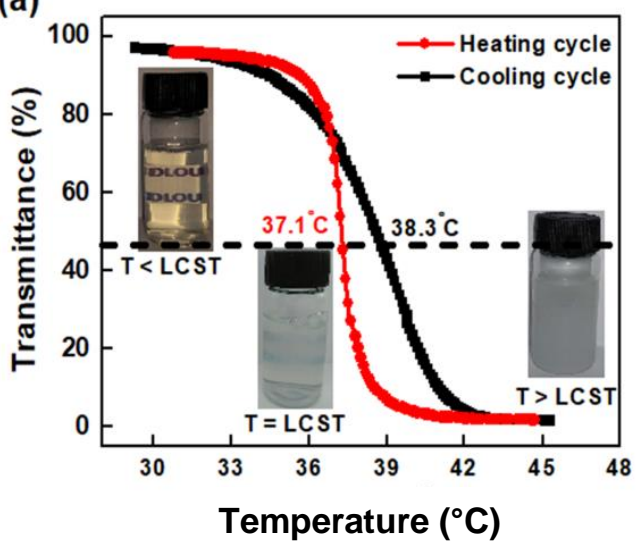

(c)

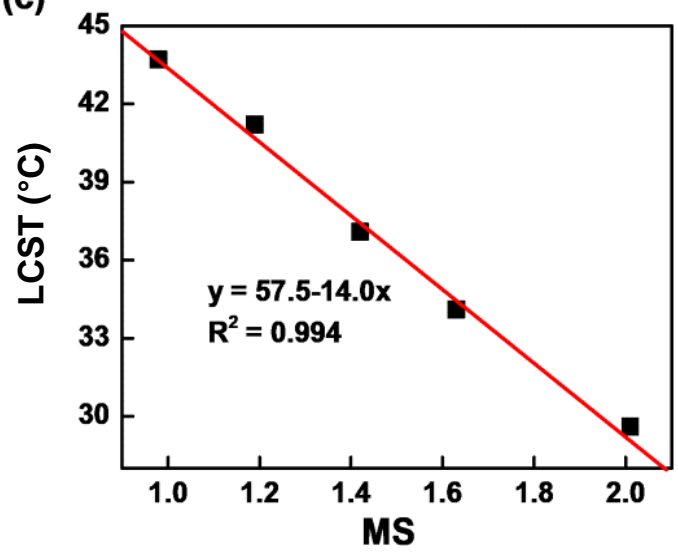

(b)

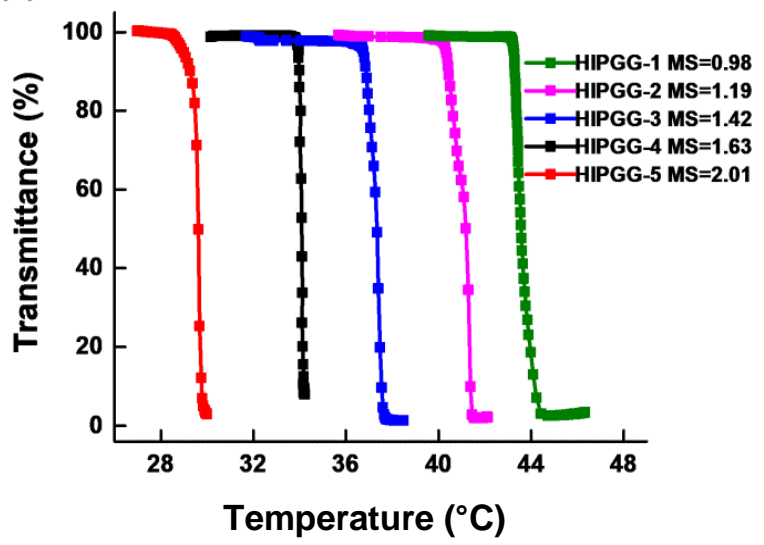

(d)

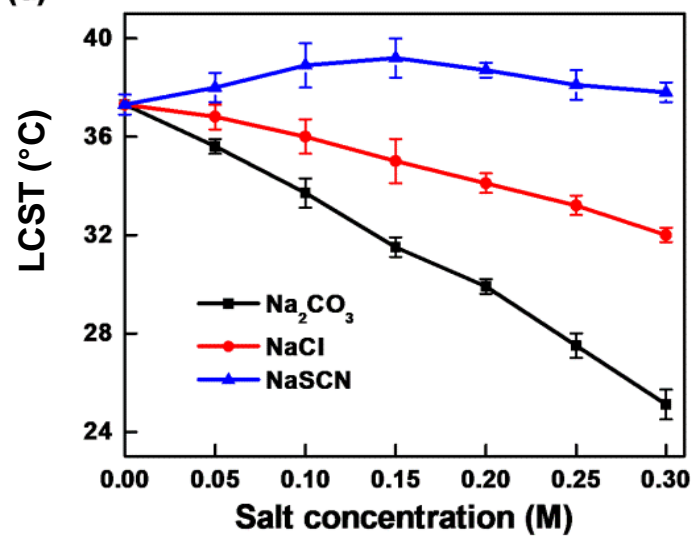

Fig. 3. (a) Plots of transmittance as a function of temperature measured for aqueous solutions $(1 \mathrm{~g} / \mathrm{L})$ of HIPGG-3 (insets show visual changes of the transmittance of sample solutions below and above the LCST); (b) Transmittance changes for aqueous solutions (1 g/L) of HIPGG-1 to HIPGG-5; (c) Plot of the measured LCSTs as a function of MS; (d) Plots of the measured LCSTs as a function of $\mathrm{Na}_{2} \mathrm{CO}_{3}, \mathrm{NaCl}$, and $\mathrm{NaSCN}$ concentrations

The aqueous solution of HIPGG-3 (1 g/L) exhibited hysteresis of $1.2{ }^{\circ} \mathrm{C}$ in the LCST during the cooling process $\left(38.3^{\circ} \mathrm{C}\right)$ relative to the heating process $\left(37.1^{\circ} \mathrm{C}\right)$ (Fig. $3 a)$. Figure $3 \mathrm{~b}$ shows the changes in transmittance for aqueous solutions of HIPGG with different MS (1 g/L) vs. temperature. The transmittance of all the HIPGG solutions with different MS decreased sharply when the temperature was increased to the LCSTs. For example, the transparent HIPGG-3 aqueous solution became turbid when the temperature was increased to $37.1{ }^{\circ} \mathrm{C}$ (Fig. 1a). This was because the hydrophilic framework (GG) of HIPGG formed hydrogen bonds with water molecules at low temperatures. Thus, HIPGG could dissolve in water. Conversely, when the temperature was increased to the LCST, the hydrogen bonds between HIPGG and water molecules were destroyed. Subsequently, the interactions between hydrophobic alkyl chains become dominant, which caused aggregation of the HIPGG molecules, and the LCST exhibited linear decreases with increases in the MS. Specifically, as seen in Fig. 3c, the LCST decreased from $43.7{ }^{\circ} \mathrm{C}$ to $29.6{ }^{\circ} \mathrm{C}$ when the MS was increased from 0.98 to 2.01. This showed that varying the MS of isopropyl glycidyl ether enabled the LCST of HIPGG to be precisely adjusted. As temperature increased, the HIPGGs with higher MS possessed stronger hydrophobic association between molecules, which led to decreases in LCST. Notably, the HIPGG 
samples that had MS values in the range of 0.98 to 2.01 had a desirable thermo-responsive property. However, when the MS was less than 0.98, the HIPGG solution did not have phase separation behavior during heating, which was because of the low number of hydrophobic side chains and the weak hydrophobicity. When the MS was greater than 2.01, HIPGG did not dissolve in water because of its excessively strong hydrophobicity.

Adding inorganic salts could also be used to adjust the LCST of HIPGG aqueous solution. Plots exhibiting the variations in transmittance with respect to the temperature at different concentrations of $\mathrm{NaSCN}, \mathrm{NaCl}$, or $\mathrm{Na}_{2} \mathrm{CO}_{3}$ are given in Fig. 3d for an HIPGG3 concentration of $1 \mathrm{~g} / \mathrm{L}$. When the concentrations of $\mathrm{NaCl}$ and $\mathrm{Na}_{2} \mathrm{CO}_{3}$ were increased from $0 \mathrm{M}$ to $0.3 \mathrm{M}$, the LCST of HIPGG decreased linearly from $37.1{ }^{\circ} \mathrm{C}$ to $34.4{ }^{\circ} \mathrm{C}$ and $27.3^{\circ} \mathrm{C}$, respectively. As mentioned above, HIPGG could be dissolved in water because of hydrogen bonding between HIPGG and water. When $\mathrm{NaCl}$ or $\mathrm{Na}_{2} \mathrm{CO}_{3}$ was added to the solution, the hydrogen bonding between HIPGG and water molecules weakened. This resulted in easier removal of water molecules from the HIPGG molecular chain during heating, which reduced the LCST. In contrast, in the presence of NaSCN, the variations of LCST were nonlinear with respect to concentration. Specifically, the LCST increased as the NaSCN concentration increased, which was followed by a decrease at larger concentrations of $\mathrm{NaSCN}$. The added $\mathrm{SCN}^{-}$complexed with the hydroxyl groups on the HIPGG molecular chain, which increased the solubility of HIPGG, thereby increasing the LCST. The presence of these three kinds of inorganic salts affected the LCSTs in accordance with the Hofmeister series (Yan and Mu 2015; Yan et al. 2016).

\section{Self-aggregation of HIPGG in Aqueous Solution}

Similar to PINPAM, amphiphilic HIPGG self-assembles into aggregates when it is at a certain concentration in water. In this study, fluorescence spectrometry with pyrene as a fluorescence probe was used to study the formation of HIPGG aggregates. The solubility of pyrene in aqueous solutions is small. However, in a hydrophobic environment, the fluorescence spectrum of pyrene changes considerably, and thus pyrene is often used to determine the CAC. Figure 4a shows the excitation spectrum of pyrene with HIPGG-3 at different concentrations. With the increase in the HIPGG-3 concentration from $0.0005 \mathrm{~g} / \mathrm{L}$ to $0.7 \mathrm{~g} / \mathrm{L}$, the intensity of the fluorescence excitation spectra of pyrene gradually increased. The maximum peak intensity of the excitation spectrum of pyrene red-shifted from $334 \mathrm{~nm}$ to $338 \mathrm{~nm}$ when the aqueous solution of HIPGG-3 reached a certain concentration. This indicated that the polarity of the environment of pyrene changed from a polar environment to a weakly polar or nonpolar environment. This also indicated that HIPGG aggregates formed and encapsulated pyrene in the hydrophobic region of the aggregates. The intensity ratio of the peaks at $338 \mathrm{~nm}$ and $334 \mathrm{~nm}\left(I_{338} / I_{334}\right)$ was calculated and plotted versus the HIPGG-3 concentrations (Fig. 4b). The sharp increase in the intensity ratio of the pyrene peaks at $338 \mathrm{~nm}$ and $334 \mathrm{~nm}$ in the excitation spectra indicated that the CAC for HIPGG-3 was approximately $0.051 \mathrm{~g} / \mathrm{L}$. In addition, the CAC values of the HIPGG samples decreased as MS increased (Fig. 4c). The HIPGG samples that had higher MS possessed stronger hydrophobicity, which caused hydrophobic interactions among HIPGG molecules to occur more easily; thus, the CAC decreased.

Dynamic light scattering and TEM were used to investigate variations in the size of the HIPGG-1 to HIPGG-5 aggregates with respect to temperature. As can be seen in Fig. $4 \mathrm{~d}$, in the lower temperature ranges, the diameters of the HIPGG-1 to HIPGG-5 aggregates were relatively small, and there were no noticeable changes. 
(a)

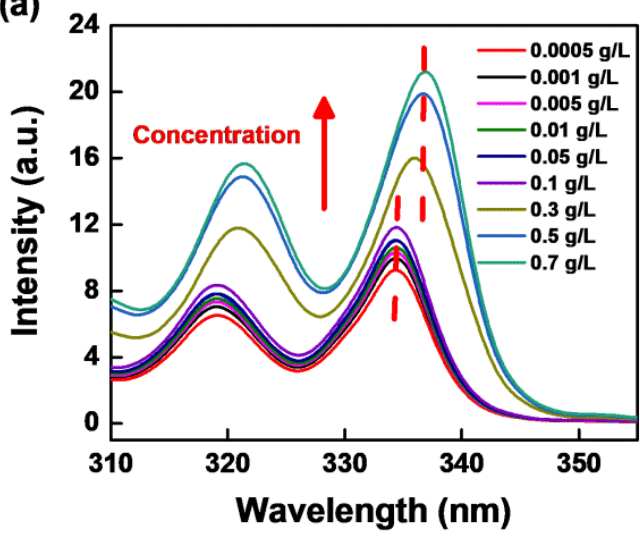

(c)

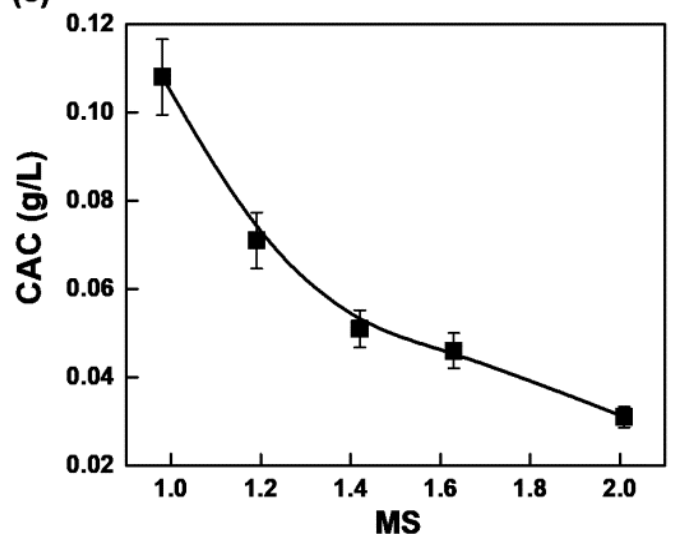

(e)

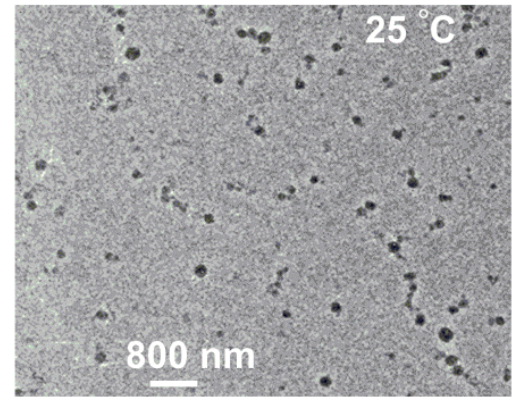

(b)

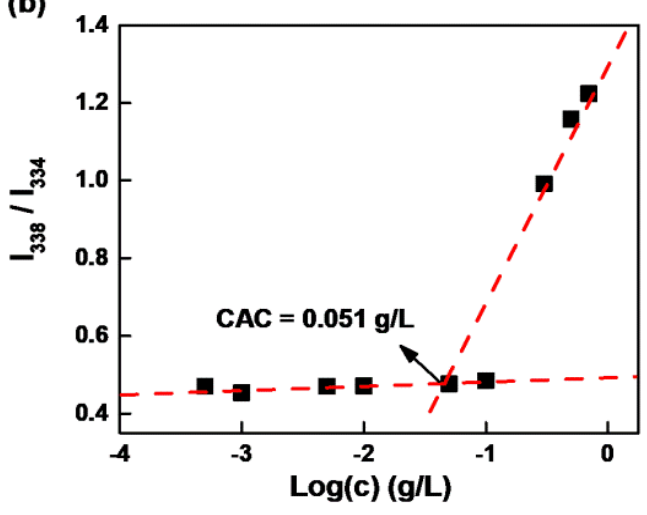

(d)
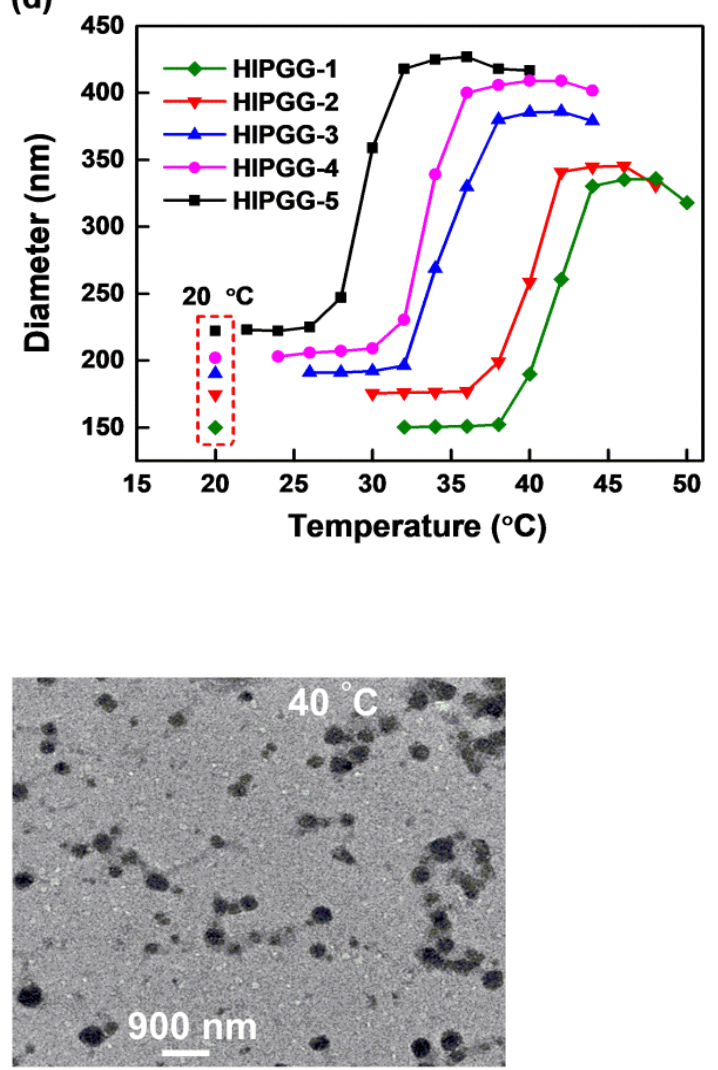

Fig. 4. (a) Excitation spectra of pyrene; (b) $/ 338 / 334$ values of aqueous solutions of HIPGG-3 at different concentrations (pyrene concentration of $6 \times 10^{-7} \mathrm{M}$ and $\lambda_{\mathrm{em}}=490 \mathrm{~nm}$ ); (c) Plots of the measured CAC as a function of MS; (d) Temperature dependence of hydrodynamic diameters for HIPGG-1 to HIPGG-5 aggregates in aqueous solutions (concentration of $1 \mathrm{~g} / \mathrm{L}$ ); (e) TEM images of HIPGG-3 aggregates at $25^{\circ} \mathrm{C}$ and $40^{\circ} \mathrm{C}$

For example, the diameter of HIPGG-3 aggregates was approximately $191 \mathrm{~nm}$ in the range of $20^{\circ} \mathrm{C}$ to $32^{\circ} \mathrm{C}$. The values of the hydrodynamic diameter of the HIPGG- 1 to HIPGG-4 aggregates were smaller than that of HIPGG-5. This was because the HIPGG samples with higher MS were more hydrophobic and thus formed a larger hydrophobic region, which resulted in an increase in the diameters of the aggregates. In contrast, the 
size of the HIPGG-1 to HIPGG-5 aggregates increased rapidly in the higher temperature ranges. For example, when the temperature was increased from $32{ }^{\circ} \mathrm{C}$ to $38^{\circ} \mathrm{C}$, the size of the HIPGG-3 aggregates increased sharply from $191 \mathrm{~nm}$ to $385 \mathrm{~nm}$. Remarkably, the size of the aggregates decreased slightly when the temperature of the solution was increased from $42{ }^{\circ} \mathrm{C}$ to $44{ }^{\circ} \mathrm{C}$, which was probably because the solutions of the aggregates further heated up as a result of dehydration and shrinkage. This result was similar to that observed for aggregates of poly(2-alkyl-2-oxazoline)s (Obeid et al. 2009).

To provide further support for the DLS results, TEM was performed to monitor changes in the morphology and size of the HIPGG aggregates upon increasing the temperature increase from $25{ }^{\circ} \mathrm{C}$ to $40{ }^{\circ} \mathrm{C}$. Figure $4 \mathrm{e}$ shows that smaller aggregates of HIPGG-3 (average diameter of approximately $176 \mathrm{~nm}$ ) were observed at $25^{\circ} \mathrm{C}$, whereas larger aggregates (average diameter of approximately $368 \mathrm{~nm}$ ) were observed at $40{ }^{\circ} \mathrm{C}$. Remarkably, the size of the aggregates that were observed via TEM at temperatures in the range of $25^{\circ} \mathrm{C}$ and $40{ }^{\circ} \mathrm{C}$ was smaller than that determined via DLS. This may have been because dehydration of the aggregates was induced by water evaporation, which resulted in the shrinkage of aggregates in the TEM experiment (Yuan et al. 2011; Ten Brummelhuis et al. 2012).

\section{Encapsulation of Nile Red and Its Temperature-controlled Release}

Fluorescence spectroscopy with Nile red as a model for a hypothetical drug was used to study the encapsulation and temperature-controlled release behaviors of the HIPGG aggregates. Nile red is mostly insoluble in water, it presents little fluorescence signal. However, the fluorescence intensity of Nile red dramatically increases when it is in a hydrophobic environment, such as when it is in the hydrophobic region of aggregates (Chen and Guan 2004). In this study, the fluorescence intensity of Nile red was investigated using photoluminescence spectroscopy with an excitation wavelength of $490 \mathrm{~nm}$ with HIPGG-3 at different concentrations. Figure 5a shows that the fluorescence intensity of Nile red was invisible in the absence of HIPGG-3 aggregates. However, there was a clear intense peak at $625 \mathrm{~nm}$ observed when HIPGG-3 was in aqueous solution. In addition, the fluorescence intensity of Nile red was relatively weak when the concentration of the HIPGG-3 solution was lower than its CAC, whereas it increased rapidly when the concentration was above its CAC. Many HIPGG-3 aggregates formed above the CAC, which provided a larger hydrophobic region for encapsulating Nile red and contributed to the increased intensity (Kim et al. 2009; Park et al. 2010). A similar tendency was also observed for other HIPGG samples that had different MS (Fig. 5b). Specifically, the fluorescence intensities at maximum values were plotted against the concentration of HIPGG-1 to HIPGG-5. For all the HIPGG samples, the fluorescence intensity at $\lambda_{\max }$ for Nile red exhibited a steady increase with respect to the concentration of samples, and there was a relatively rapid increase observed when the concentration was above the CAC.

The encapsulation of Nile red in HIPGG aggregates could be controllably released in response to various temperatures. The release of Nile red from HIPGG aggregates was examined using fluorescence spectrometry because the fluorescence intensity of Nile red decreased dramatically in aqueous solution compared to that in the hydrophobic region of the HIPGG aggregates. Thus, the fluorescence intensity of Nile red that was encapsulated in HIPGG aggregates was measured over time at $36{ }^{\circ} \mathrm{C}, 38{ }^{\circ} \mathrm{C}$, and $42^{\circ} \mathrm{C}$. Figure $5 \mathrm{c}$ shows that the temperature control of the release behavior of the aggregates. 
(a)

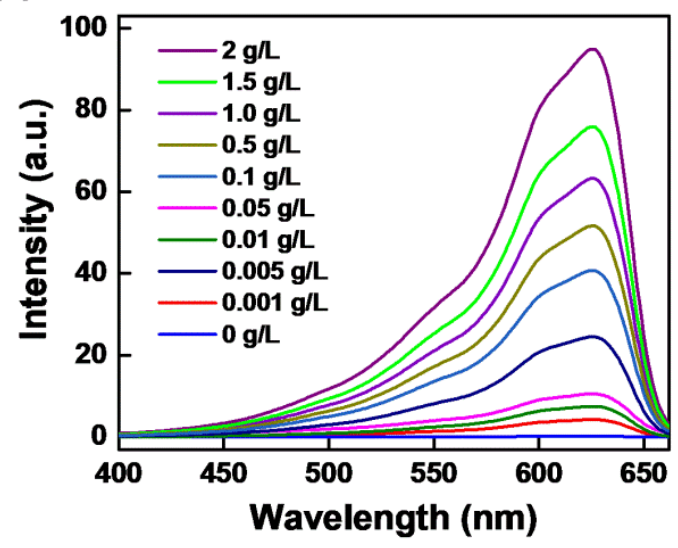

(b)

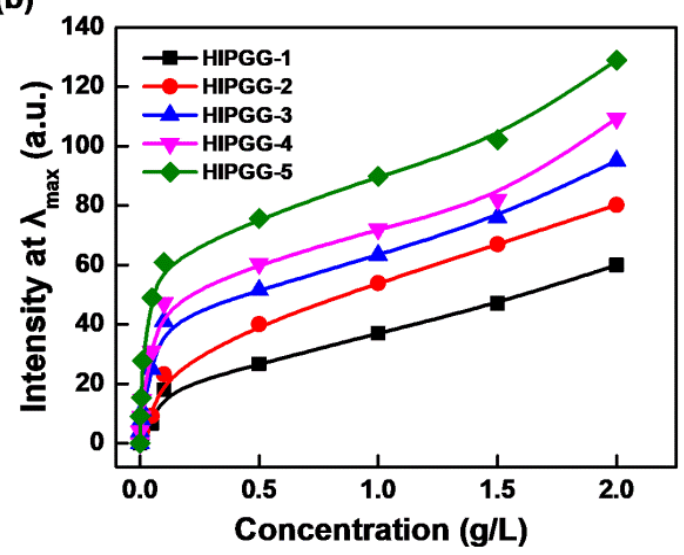

(c)

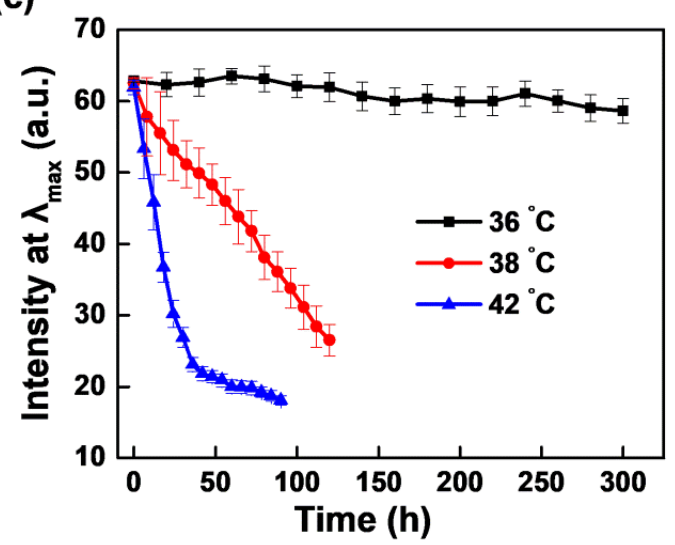

Fig. 5. (a) Emission spectra for Nile red in aqueous solutions with different concentrations of HIPGG-3 $\left(\lambda_{\text {ex }}=490 \mathrm{~nm}\right.$ ); (b) Fluorescence intensity at $\lambda_{\max }$ for the emission spectra of Nile red as a function of concentration of HIPGG-1 to HIPGG-5; (c) Nile red fluorescence in the aggregates of HIPGG-3 as a function of time at $36^{\circ} \mathrm{C}, 38^{\circ} \mathrm{C}$, and $42{ }^{\circ} \mathrm{C}$

The fluorescence intensity of Nile red in HIPGG-3 aggregates did not obviously decrease during incubation for $300 \mathrm{~h}$ at $36^{\circ} \mathrm{C}$, it was indicated that Nile red could be stably present in HIPGG aggregates when the temperature was lower than LCST. However, the intensity of Nile red gradually decreased during incubation for $120 \mathrm{~h}$ at $38{ }^{\circ} \mathrm{C}$ (LCST = $37.1^{\circ} \mathrm{C}$ ). When the temperature was higher than the LCST of HIPGG, the hydrophilic shell collapsed. This resulted in deformation of the aggregate structure, and the Nile red was released from the hydrophobic region to the aqueous solution, which led to the fluorescence quenching of Nile red. In addition, changing the temperatures enabled the encapsulated guest molecules to be easily and controllably released from HIPGG aggregates (Fig. 5c), and the fluorescence intensity decreased more rapidly at higher temperatures. Interestingly, at $42{ }^{\circ} \mathrm{C}$, the fluorescence intensity remained constant after $50 \mathrm{~h}$, and no further obvious decrease in the fluorescence intensity of Nile red was monitored. This was in line with the results of other amphiphilic thermo-responsive polymers (Bose et al. 2017). Nile red remained encapsulated in the collapsed HIPGG-3 aggregates because of shrinkage at 42 ${ }^{\circ} \mathrm{C}$ to $44{ }^{\circ} \mathrm{C}$. 


\section{CONCLUSIONS}

1. In this work, a novel guar gum-based thermo-responsive polymer (HIPGG) was synthesized via etherification reaction, and it had a tunable lower critical solution temperature (LCST). Changing the molar substitution (MS) of the hydrophobic chain enabled the LCST of HIPGG to be tuned. Specifically, when the MS of HIPGG increased from 0.98 to 2.01 , the LCST decreased from $43.7^{\circ} \mathrm{C}$ to $29.6{ }^{\circ} \mathrm{C}$. In addition, inorganic salts $\left(\mathrm{NaSCN}, \mathrm{NaCl}\right.$, and $\left.\mathrm{Na}_{2} \mathrm{CO}_{3}\right)$ had a remarkable effect on the LCST of HIPGG.

2. Fluorescence spectroscopy, DLS, and TEM were used to research the self-assembly behavior and thermo-responsive property of HIPGG aggregates. Investigations showed that temperature changes could be used to change the size and structure of the HIPGG aggregates.

3. The behaviors of encapsulation and release of Nile red in HIPGG aggregates were investigated at temperatures above and below the LCST. At temperatures below the LCST of HIPGG, the Nile red was stably encapsulated in HIPGG aggregates, whereas it was released from aggregates when the temperature was higher than the LCST. Additionally, changing the temperature of the aqueous solution of HIPGG-Nile red enabled control of the release rate of Nile red. In brief, HIPGG aggregates with desirable thermo-responsive properties have broad application prospects in drug release platforms and in other appropriate biological research.

\section{ACKNOWLEDGMENTS}

The authors are grateful for the support of the National Key R\&D Program of China (No. 2017YFD0701700), the National Natural Science Foundation of China (No. 31901775 and No. 31672673), the Key R\&D Program of Guangdong Province (No. 2019B020215001), and the Liaoning S\&T Project (No. 2017203002).

\section{REFERENCES CITED}

Alejo, T., Uson, L., and Arruebo, M. (2019). "Reversible stimuli-responsive nanomaterials with on-off switching ability for biomedical applications," Journal of Controlled Release 314, 162-176. DOI: 10.1016/j.jconrel.2019.10.036

Atanase, L. I., Desbrieres, J., and Riess, G. (2017). "Micellization of synthetic and polysaccharides-based graft copolymers in aqueous media," Progress in Polymer Science 73, 32-60. DOI: 10.1016/j.progpolymsci.2017.06.001

Bordat, A., Boissenot, T., Nicolas, J., and Tsapis, N. (2019). "Thermoresponsive polymer nanocarriers for biomedical applications," Advanced Drug Delivery Reviews 138, 167-192. DOI: 10.1016/j.addr.2018.10.005

Bose, A., Jana, S., Saha, A., and Mandal, T. K. (2017). “Amphiphilic polypeptidepolyoxazoline graft copolymer conjugate with tunable thermoresponsiveness: Synthesis and self-assembly into various micellar structures in aqueous and nonaqueous media," Polymer 110, 12-24. DOI: 10.1016/j.polymer.2016.12.068 
Carter, S., Rimmer, S., Rutkaite, R., Swanson, L., Fairclough, J. P. A., Sturdy, A., and Webb, M. (2006). "Highly branched poly( $N$-isopropylacrylamide) for use in protein purification," Biomacromolecules 7(4), 1124-1130. DOI: 10.1021/bm050929h

Chen, G. H., and Guan, Z. B. (2004). "Transition metal-catalyzed one-pot synthesis of water-soluble dendritic molecular nanocarriers," Journal of the American Chemical Society 126(9), 2662-2663. DOI: 10.1021/ja039829e

Dai, M., Liu, Y., Ju, B., and Tian, Y. (2019a). "Preparation of thermoresponsive alginate/starch ether composite hydrogel and its application to the removal of $\mathrm{Cu}$ (II) from aqueous solution," Bioresource Technology 294, Article ID 122192. DOI: 10.1016/j.biortech.2019.122192

Dai, M., Tian, Y., Fan, J., Ren, J., Liu, Y., Rahman, M., Ju, B., Ren, X., and Ma, H. (2019b). "Tuning of lower critical solution temperature of thermoresponsive 2hydroxy-3-alkoxypropyl hydroxyethyl cellulose by alkyl side chains and additives," BioResources 14(4), 7977-7991. DOI: 10.15376/biores.14.4.7977-7991

Graham, S., Marina, P. F., and Blencowe, A. (2019). "Thermoresponsive polysaccharides and their thermoreversible physical hydrogel networks," Carbohydrate Polymers 207, 143-159. DOI: 10.1016/j.carbpol.2018.11.053

Grzelczak, M., Liz-Marzán, L. M., and Klajn, R. (2019). "Stimuli-responsive selfassembly of nanoparticles," Chemical Society Reviews 48(5), 1342-1361. DOI: $10.1039 / \mathrm{c} 8 \mathrm{cs} 00787 \mathrm{j}$

Gupta, N. R., Ghute, P. P., and Badiger, M. V. (2011). "Synthesis and characterization of thermosensitive graft copolymer of carboxymethyl guar and poly( $N$-isopropylacrylamide)," Carbohydrate Polymers 83(1), 74-80. DOI: 10.1016/j.carbpol.2010.07.023

Gupta, N. R., Torris, A. A. T., Wadgaonkar, P. P., Rajamohanan, P. R., Ducouret, G., Hourdet, D., Creton, C., and Badiger, M. V. (2015). "Synthesis and characterization of PEPO grafted carboxymethyl guar and carboxymethyl tamarind as new thermoassociating polymers," Carbohydrate Polymers 117, 331-338. DOI: 10.1016/j.carbpol.2014.09.073

Kim, S. H., Tan, J. P. K., Nederberg, F., Fukushima, K., Yang, Y. Y., Waymouth, R. M., and Hedrick, J. L. (2009). "Mixed micelle formation through stereocomplexation between enantiomeric poly(lactide) block copolymers," Macromolecules 42(1), 2529. DOI: $10.1021 / \mathrm{ma} 801739 x$

Nagase, K., Yamato, M., Kanazawa, H., and Okano, T. (2018). "Poly(N-isopropylacrylamide)-based thermoresponsive surfaces provide new types of biomedical applications," Biomaterials 153, 27-48. DOI: 10.1016/j.biomaterials.2017.10.026

Obeid, R., Maltseva, E., Thünemann, A. F., Tanaka, F., and Winnik, F. M. (2009). "Temperature response of self-assembled micelles of telechelic hydrophobically modified poly(2-alkyl-2-oxazoline)s in water," Macromolecules 42(6), 2204-2214. DOI: $10.1021 / \mathrm{ma} 802592 \mathrm{f}$

Park, J., Moon, M., Seo, M., Choi, H., and Kim, S. Y. (2010). "Well-defined star-shaped rod-coil diblock copolymers as a new class of unimolecular micelles: Encapsulation of guests and thermoresponsive phase transition," Macromolecules 43(19), 83048313. DOI: $10.1021 / \mathrm{ma101567p}$

Prabaharan, M. (2011). "Prospective of guar gum and its derivatives as controlled drug delivery systems," International Journal of Biological Macromolecules 49(2), 117 124. DOI: 10.1016/j.ijbiomac.2011.04.022 
Roy, D., Brooks, W. L. A., and Sumerlin, B. S. (2013). "New directions in thermoresponsive polymers," Chemical Society Reviews 42(17), 7214-7243. DOI: $10.1039 / \mathrm{c} 3 \operatorname{cs} 35499 \mathrm{~g}$

Shen, L., and Patel, M. K. (2008). "Life cycle assessment of polysaccharide materials: A review," Journal of Polymers and the Environment 16, 154-167. DOI: 10.1007/s10924-008-0092-9

Ten Brummelhuis, N., Secker, C., and Schlaad, H. (2012). "Hofmeister salt effects on the LCST behavior of poly(2-oxazoline) star ionomers," Macromolecular Rapid Communications 33(19), 1690-1694. DOI: 10.1002/marc.201200406

Tian, Y., Ju, B., Zhang, S., and Hou, L. (2016). "Thermoresponsive cellulose ether and its flocculation behavior for organic dye removal," Carbohydrate Polymers 136, 1209 1217. DOI: 10.1016/j.carbpol.2015.10.031

Wang, C., Yang, T., Wang, T., and Qiu, L. (2018). "Thermosensitive behavior of hydrophobically associating anionic guar gum solutions and gels," International Journal of Biological Macromolecules 111, 169-177. DOI:

10.1016/j.ijbiomac.2018.01.017

Wu, W., Li, J., Liu, W., and Deng, Y. (2016). “Temperature-sensitive, fluorescent poly(N-isopropyl-acrylamide)-grafted cellulose nanocrystals for drug release," Bioresources 11(3), 7026-7035. DOI: 10.15376/biores.11.3.7026-7035

Yan, C., and Mu, T. (2015). "Molecular understanding of ion specificity at the peptide bond," Physical Chemistry Chemical Physics 17(5), 3241-3249. DOI: $10.1039 / \mathrm{c} 4 \mathrm{cp} 04055 \mathrm{~d}$

Yan, C., Xue, Z., Zhao, W., Wang, J., and Mu, T. (2016). "Surprising Hofmeister effects on the bending vibration of water," ChemPhysChem 17(20), 3309-3314. DOI: 10.1002/cphc.201600551

Yuan, W., Li, X., Gu, S., Cao, A., and Ren, J. (2011). "Amphiphilic chitosan graft copolymer via combination of ROP, ATRP and click chemistry: Synthesis, selfassembly, thermosensitivity, fluorescence, and controlled drug release," Polymer 52(3), 658-666. DOI: 10.1016/j.polymer.2010.12.052

Yuba, E. (2020). "Development of functional liposomes by modification of stimuliresponsive materials and their biomedical applications," Journal of Materials Chemistry B 8(6), 1093-1107. DOI: 10.1039/c9tb02470k

Article submitted: June 15, 2020; Peer review completed: August 8, 2020; Revised version received and accepted: August 14, 2020; Published: August 19, 2020.

DOI: $10.15376 /$ biores. 15.4.7615-7627 